Revista Ambientale

Revista da Universidade Estadual de Alagoas/UNEAL

e-ISSN 2318-454X, Ano 13, Vol. 13 (4), 2021

https://doi.org/10.48180/ambientale.v13i4.319

\title{
Análise espacial de similaridade da qualidade da água de um rio localizado no sul do Rio Grande do Sul
}

\section{Spatial analysis of the similarity of the water quality of a river located in the south of Rio Grande do Sul}

\begin{abstract}
Gisele Silva de Souza ${ }^{1}$, Samanta Tolentino Cecconello ${ }^{2}$, Luana Nunes Centeno ${ }^{3}$
1. Instituto Federal de Educação, Ciência e Tecnologia Sul-rio-grandense Câmpus Pelotas, email: zeka.ss@ @otmail.com; 2. Instituto Federal de educação, Ciência e Tecnologia Sul-riograndense Câmpus Pelotas, e-mail: samantacecconello@ifsul.edu.br; 3. Universidade Federal de Pelotas, e-mail: luananunescenteno@gmail.com
\end{abstract}

E-mail do autor correspondente: zeka.ss@ hotmail.com

Resumo - A qualidade da água de um manancial está intimamente relacionada as suas características físicas-químicas e biológicas e suas interações, são funções dos seus diversos usos. Diante disto, este trabalho objetivou analisar a inter-relação entre pontos de monitoramento da qualidade da água pertencentes ao Rio Comandaí, localizado na bacia hidrográfica do Uruguai, bem como inferir sobre suas possíveis fontes de poluição. Foram utilizados cinco pontos de monitoramento da qualidade da água no Rio Comandaí, sendo que em cada ponto foram utilizados 14 parâmetros de qualidade da água, disponibilizados pela FEPAM. Neste estudo empregou-se a análise de agrupamento utilizando o método de Ward's e como medida de similaridade utilizou-se a distância Euclidiana. Com base neste estudo foi possível observar que os cinco pontos encontraram resultados distintos para as possíveis fontes de poluição, mesmo tendo estabelecido o mesmo ponto de corte na similaridade (80\%). Isso demonstra que ao longo do rio Comandaí, há uma alteração no uso e coberturas da terra o que modifica as fontes de poluição. Sendo assim, foi possível inferir sobre as possíveis fontes de poluição no rio Comandaí, bem como a similaridade entre as possíveis fontes de poluição.

Palavras-Chaves: Rio Comandaí. Análise de cluster. Fontes de poluição.

\footnotetext{
Abstract - The quality of water in a source is closely related to its physical-chemical and biological characteristics and their interactions, which are functions of its various uses. Therefore, this work aimed to analyze the interrelationship between water quality monitoring points belonging to the Comandaí River, located in the Uruguayan hydrographic basin, as well as to infer about its possible sources of pollution. Five water quality monitoring points were used on the Comandaí River, and at each point 14 water quality parameters were used, provided by FEPAM. In this study, cluster analysis was used using the Ward's method and the Euclidean distance was used as a measure of similarity. Based on this study, it was possible to observe that the five points found different results for the possible sources of pollution, even having established the same cut-off point for similarity (80\%). This demonstrates that along the Comandaí River, there is a change in land use and land cover, which modifies the sources of pollution. Thus, it was possible to infer about the possible sources of pollution in the Comandaí River, as well as the similarity between the possible sources of pollution.
} 


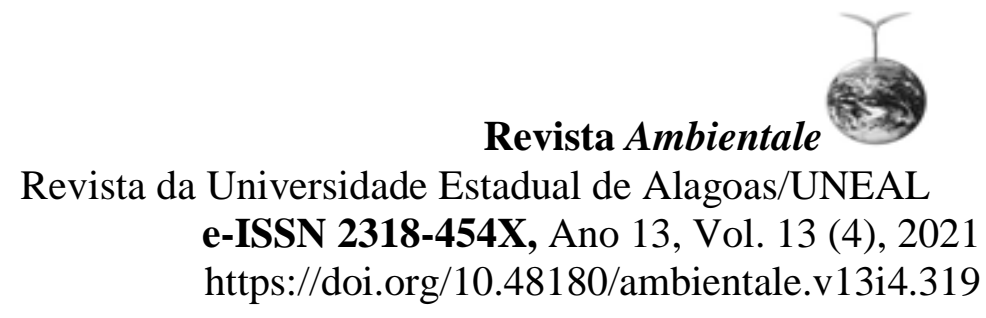

Keywords: Comandaí River. Cluster analysis. Sources of pollution.

\section{Introdução}

A água é um recurso natural renovável e finito (GLORIA; HORN; HILGEMANN, 2017) o que torna o seu monitoramento e gestão, de suma importância (ZAMBERLAN et al., 2013). Entretanto, a qualidade dos recursos hídricos, é fortemente influenciada pelos processos de urbanização, pelas indústrias e também pelas atividades agropecuárias (SANTOS et al., 2017).

Neste contexto, o monitoramento da qualidade da água possibilita avaliar sua qualidade (TRINDADE et al., 2016), com o intuito de garantir a continuidade e assegurar uma boa gestão dos recursos hídricos (UNCUMUSAOĞLU; AKKAN, 2017). Dentre os órgãos e agências reguladoras responsáveis pelo manejo adequado e monitoramento dos recursos hídricos, tema Agência Nacional de Águas (ANA, 2007), o Programa Nacional de Avaliação da Qualidade das Águas (PNQA), a Rede Nacional de Monitoramento da Qualidade de Água (RNQA) e o Programa de Estímulo à Divulgação de Dados de Qualidade de Água (Qualiágua). Ademais, se têm a Resolução do CONAMA n ${ }^{\circ} 357$, de 17 de março de 2005, que estabelece a classificação e enquadramento dos corpos d'água no Brasil (BRASIL, 2005). Este enquadramento considera como parâmetros as variáveis físico-químicas e biológicas dos corpos hídricos.

Todavia, para realização de monitoramento e gestão de um manancial é essencial obterse uma visão global do mesmo, necessário assim é, a utilização de ferramentas estatísticas capazes de analisar em conjunto, diversos parâmetros de qualidade da água (GOMES et al.,2017). Dentre as ferramentas atualmente empregadas com esta finalidade tem-se a estatística multivariada (DAS; NAG, 2015; FERCHICHI et al.,2017; ISLAM et al.,2017; URBAN; MANFRÉ; SILVA,2018; MAGESH; CHANDRASEKAR; ELANGO, 2017; ZHANG et al.,2018). A estatística multivariada é uma técnica que sintetiza múltiplas variáveis, fazendo a associação entre os dados em análise, identificando por exemplo, elementos similares que interferem no corpo hídrico (COSTA, 2018).

Portanto, a utilização da estatística multivariada no estudo da qualidade da água é uma ferramenta viável, para o manejo e compreensão das informações coletadas; separação das representações de maior relevância em cada amostra em análise; para determinar a similaridade entre variáveis limnológicas de um manancial; bem como para auxiliar na diminuição de tempo e custos com análises laboratoriais (GOMES et al., 2017).

Dentre as técnicas de estatísticas multivariada tem-se a análise de agrupamento (AA) também chamada de análise hierárquica de agrupamentos (AHA) ou clusters, que é uma ferramenta utilizada com o propósito de agrupar itens similares (VICINI; SOUZA, 2005; FÁVERO; BELFIORE, 2015). As semelhanças entre as variáveis é que formam os grupos e é definida por meio de medidas de proximidades, que agrega medidas de similaridade, ou as de dissimilaridade como por exemplo a distância Euclidiana (CENTENO et al., 2016). Em cada grupo constituído na AA, busca-se obter a maior homogeneidade possível dentro dos grupos e, por conseguinte, alta heterogeneidade entre os grupos formados (HAIR et al., 2009).

Frente ao exposto este trabalho objetivou analisar a inter-relação entre pontos de monitoramento da qualidade da água pertencentes ao Rio Comandaí, localizado na bacia hidrográfica do Uruguai, bem como inferir sobre suas possíveis fontes de poluição. 
Revista Ambientale

Revista da Universidade Estadual de Alagoas/UNEAL

e-ISSN 2318-454X, Ano 13, Vol. 13 (4), 2021

https://doi.org/10.48180/ambientale.v13i4.319

\section{Material e Métodos}

\section{Caracterização da área}

De acordo com o Decreto $\mathrm{n}^{\circ}$ 53.885, de 16 de janeiro de 2017, para fins de gestão dos recursos hídricos, a Região Hidrográfica do Uruguai foi subdividida em 11 Bacias Hidrográficas, na qual está inserida a Bacia Hidrográfica U030, que é denominada Bacia Hidrográfica dos Rios Turvo-Santa Rosa- Santo Cristo (BHTSR), onde se encontra o Rio Comandaí, objeto deste estudo (RIO GRANDE DO SUL, 2018).

Segundo a Secretária do Ambiente e Desenvolvimento Sustentável (SEMA, 2019), a bacia hidrográfica em questão, situa-se norte-noroeste do Estado do Rio Grande do Sul, entre as coordenadas geográficas $27^{\circ} 07^{\prime}$ a $28^{\circ} 13^{\prime}$ de latitude Sul e $53^{\circ} 24^{\prime}$ a $55^{\circ} 20^{\prime}$ de longitude Oeste. Compreende uma área de $11.056,23 \mathrm{~km}^{2}$, no qual se destaca os rios Turvo, Santa Rosa, Santo Cristo, Amandaú e Comandai (FEPAM, 2019). A Figura 1 apresenta a localização da área de estudo.

Figura 1. Mapa de caracterização da área de estudo.

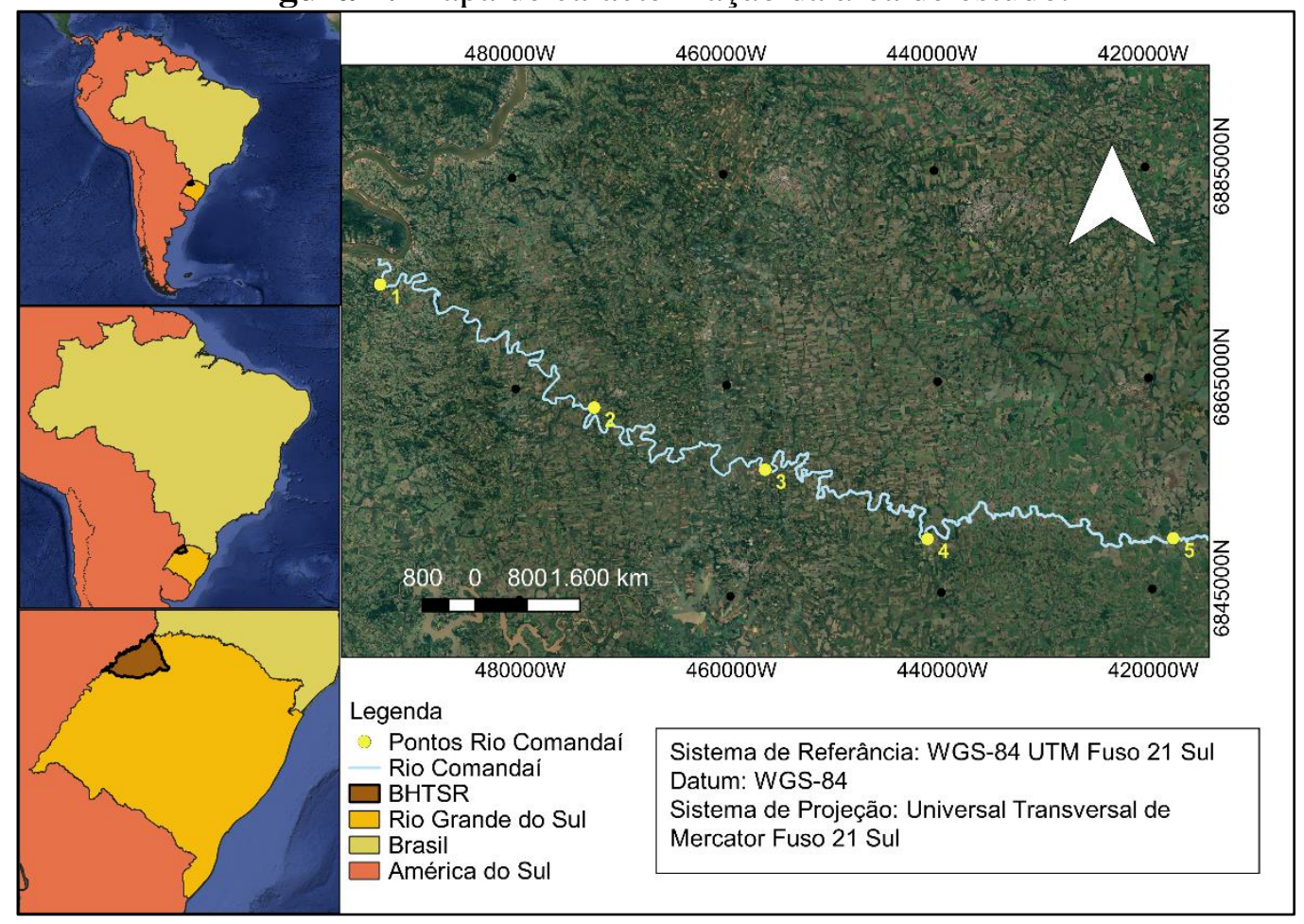

Fonte: Elaborado pelos autores.

O rio Comandaí, estende-se por $170 \mathrm{~km}$, sua nascente está situada no município de Giruá e sua foz no rio Uruguai, sua área de contribuição engloba 16 municípios (SEMA, 2019). Por ser uma região predominantemente rural, as principais fontes de contaminação na bacia hidrográfica têm sua origem nas atividades agrícola, com destaque para o plantio de soja, trigo e milho e a pecuária com ênfase para a suinocultura (FEPAM, 2019). Juntamente com as 
Revista Ambientale

Revista da Universidade Estadual de Alagoas/UNEAL

e-ISSN 2318-454X, Ano 13, Vol. 13 (4), 2021

https://doi.org/10.48180/ambientale.v13i4.319

atividades agropecuárias, as deficiências em saneamento básico dos municípios que integram a área contribuem para o aumento da carga orgânica (SEMA, 2019).

\section{Obtenção das variáveis limnológicas}

Os dados utilizados nesse estudo são dados disponibilizados pela FEPAM, e compreendem o período de 2005 até 2011. Foram realizadas coletas e análise de 23 parâmetros de qualidade da água do Rio Comandaí a saber: Temperatura da Água $\left(\mathrm{TH}_{2} \mathrm{O}\right)$, Temperatura do $\operatorname{Ar}\left(\mathrm{T}_{\mathrm{AR}}\right)$, Condutividade Elétrica $(\mathrm{CE})$, Potencial Hidrogeniônico $(\mathrm{pH})$ e Oxigênio Dissolvido (OD), em laboratório, Cloreto $\left(\mathrm{Cl}^{-}\right)$, Demanda Bioquímica de Oxigênio $\left(\mathrm{DBO}_{5}{ }^{20}\right.$ ), Demanda Química de Oxigênio (DQO), Fósforo Total (PT), Nitrato $\left(\mathrm{NO}_{3}{ }^{-}\right)$, Nitrito $\left(\mathrm{NO}_{2}{ }^{-}\right)$, Nitrogênio Kjeldahl Total (NTK), Fosfato Orto $\left(\mathrm{PO}_{4}{ }^{{ }^{3-}}\right)$, Sólidos Totais (ST), Turbidez (TH), Teores Totais de Alumínio (Al), Cobre (Cu), Ferro (Fe), Manganês (Mn), Zinco (Zn), Sódio (Na), Potássio (K) e Coliformes Termotolerantes (CT). Os métodos analíticos, bem como a preservação das amostras seguiram os procedimentos definidos pelo Standard Methods (APHA, 2005).

Todavia, em função do número de falhas amostrais, decorrentes de equipamentos em manutenção neste estudo foram utilizados 14 parâmetros de qualidade da água.

\section{Análise de agrupamento}

Neste estudo empregou-se a análise de agrupamento utilizando o método de Ward's, para ligação entre os grupos formados baseado na homogeneidade dos mesmos (DAS et al., 2018), como medida de similaridade entre as variáveis utilizou-se a distância Euclidiana (GOMES et al., 2017), medida que é frequentemente adotada na literatura científica (ESMAEILI et al., 2018). Todavia, é pertinente destacar que o método de Ward's é o único entre os métodos de agrupamento aglomerativos que se baseia em um critério clássico de soma dos quadrados, além disso, o método destaca-se dos demais, pois procura grupos no espaço euclidiano multivariado (MURTAGH; LEGENDRE, 2014). Para a realização da análise de agrupamento foi utilizado o software GENES (CRUZ, 2013).

\section{Resultados e discussão}

As variáveis de qualidade das águas coletadas e monitoradas no rio Comandaí, foram submetidas à análise de agrupamento, no qual permitiu separá-las em grupos análogos. Com isso, foram elaborados dendrogramas para cada um dos cinco pontos estudados.

A Figura 2 apresenta o dendrograma obtido a partir do monitoramento das variáveis de qualidade da água no ponto 1 do rio Comandaí, por meio de análise de agrupamento. 
Revista Ambientale

Revista da Universidade Estadual de Alagoas/UNEAL

e-ISSN 2318-454X, Ano 13, Vol. 13 (4), 2021

https://doi.org/10.48180/ambientale.v13i4.319

Figura 2. Dendrograma obtido por meio de análise de agrupamento das varáveis de qualidade de água no rio Comandaí durante o monitoramento no ponto 1.

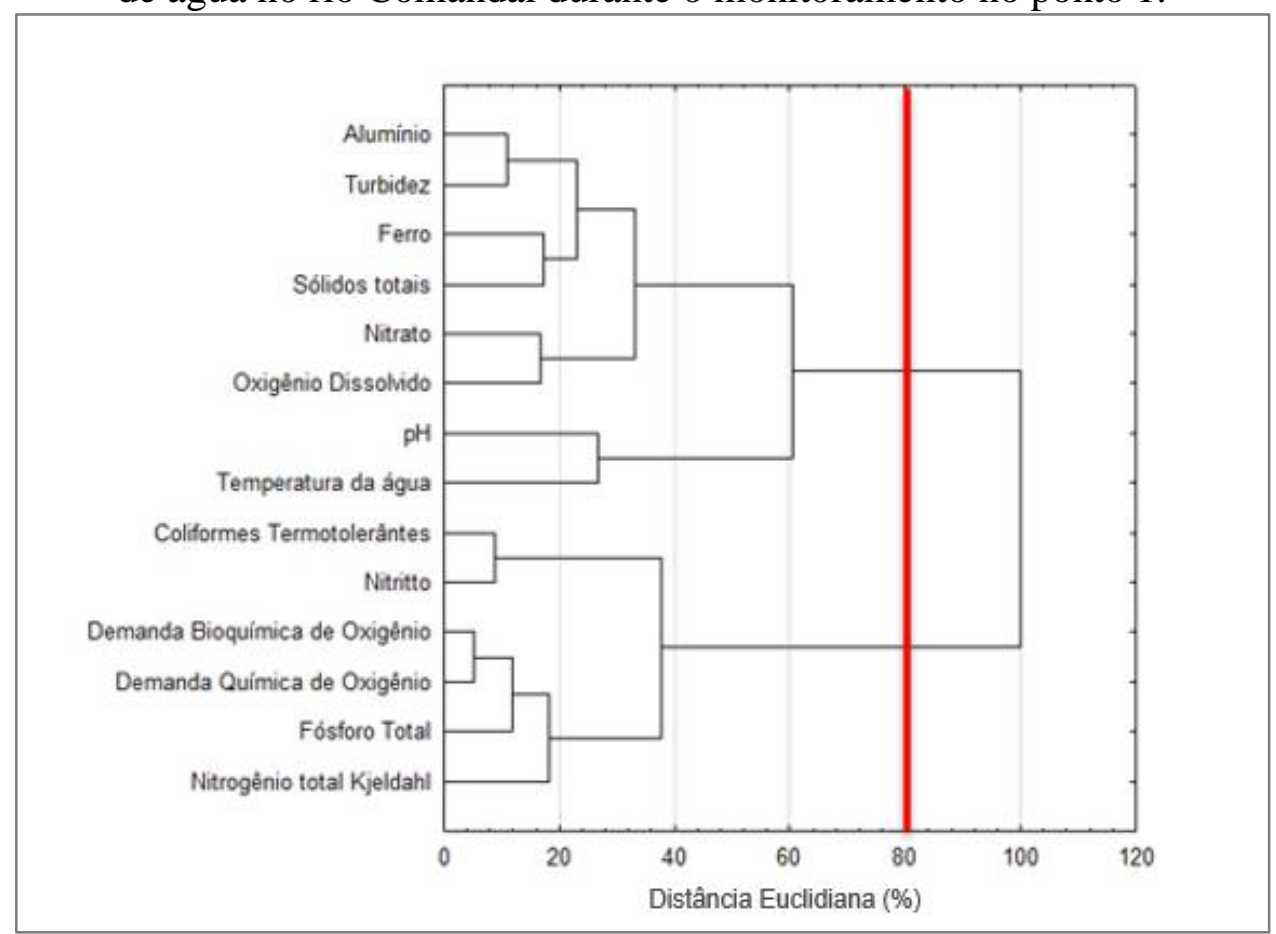

Fonte: Elaborado pelos autores.

Com base na Figura 2 o corte do dendrograma foi realizado a $80 \%$, sendo que para este e para os demais dendrogramas, seguiu-se a metodologia de Hair et al., (2009). Sendo assim, para o ponto 1 houve a formação de dois grupos. O primeiro sendo composto pelas variáveis: alumínio, turbidez, ferro, sólidos totais, nitrato, oxigênio dissolvido, $\mathrm{pH}$ e temperatura da água. Pode-se inferir que este grupo possivelmente tenha origem na dissolução de rochas, bem como pode ser oriundo de atividade agrícolas.

Por conseguinte, o grupo 2 formado tem como componentes os coliformes termotolerantes, nitrito, DBO, DQO, fósforo total e nitrogênio total Kjeldahl. Sendo que sua origem está mais associada a despejos domésticos e a pecuária.

Quando analisado o dendrograma formado com o ponto 2 (Figura 3), observa-se que este apresentou-se distinto ao dendrograma do ponto 1. 
Revista Ambientale

Revista da Universidade Estadual de Alagoas/UNEAL

e-ISSN 2318-454X, Ano 13, Vol. 13 (4), 2021

https://doi.org/10.48180/ambientale.v13i4.319

Figura 3. Dendograma obtido por meio de análise de agrupamento das varáveis de qualidade de água no rio Comandaí durante o monitoramento no ponto 2.

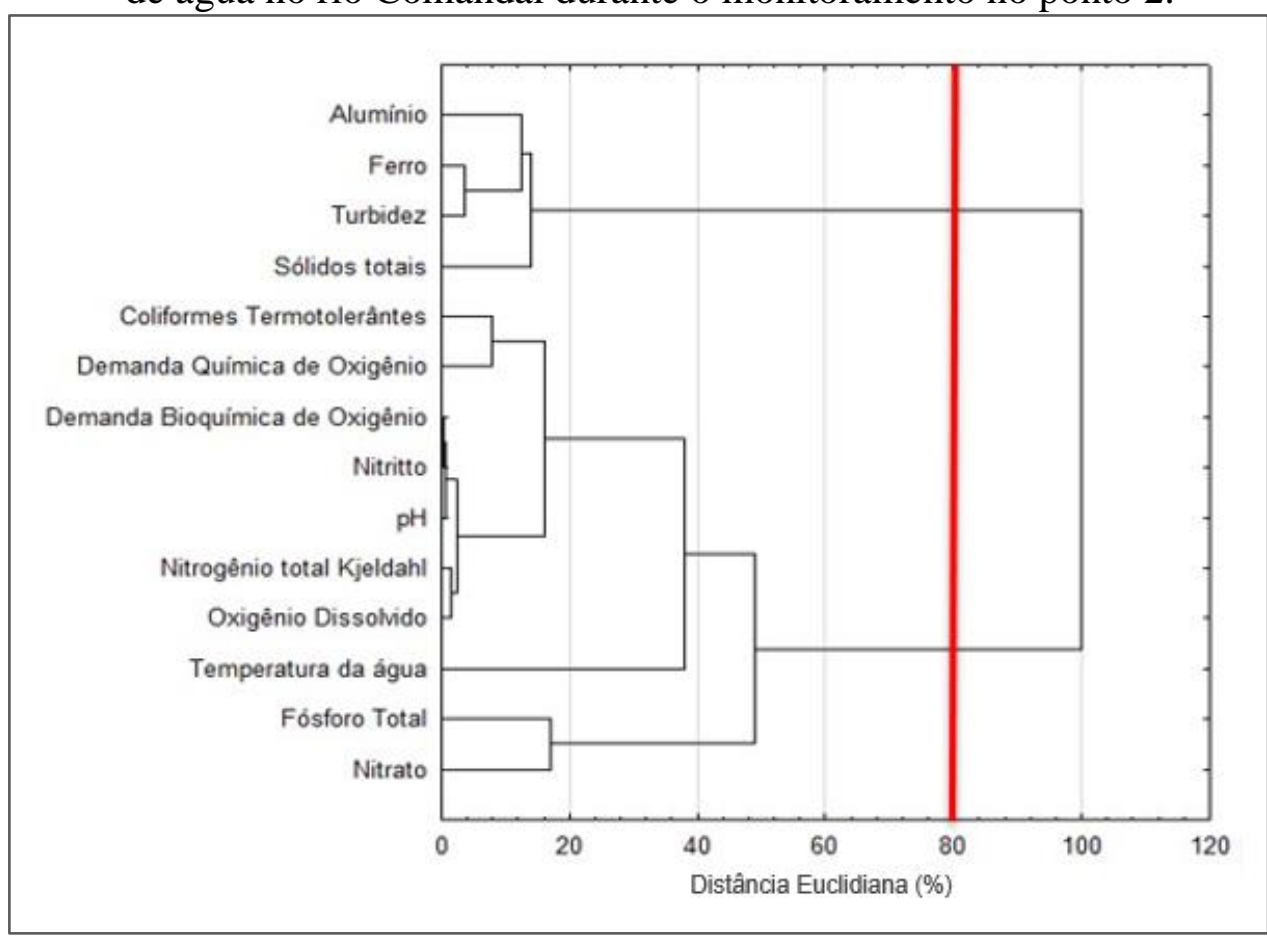

Fonte: Elaborado pelos autores.

Observa-se que no ponto 2 ocorreu a formação de dois grupos distintos, sendo que o primeiro grupo foi o alumínio, ferro, turbidez e sólidos totais, sendo possível inferir que este pode ter sua origem nos processos erosivos, assim como da dissolução de rochas e movimentação do fundo do manancial decorrente do trafego de embarcações. Já o grupo 2, possui coliformes termotolerantes, demanda bioquímica de oxigênio, demanda química de oxigênio, nitrito, $\mathrm{pH}$, nitrogênio total Kjeldahl, oxigênio dissolvido, temperatura da água, fósforo total e nitrato. O que possivelmente pode estar associado a fontes difusas como as atividades agropecuárias, das quais são representativas na região estudada.

No ponto 3, conforme demonstra a Figura 4, foram formados dos dois grupos. O primeiro grupo foi composto pelas variáveis alumínio, sólidos totais, turbidez, demanda química de oxigênio, fósforo total, $\mathrm{pH}$, nitrato, oxigênio dissolvido, demanda bioquímica de oxigênio e ferro. Possivelmente pode-se associar a este grupo as fontes de poluição oriundas das atividades agrícolas e ao uso e manejo do solo. Já o segundo grupo foi formado por quatro parâmetros de qualidade da água sendo eles coliformes termotolerantes, nitrito, nitrogênio Kjeldahl e temperatura da água, sendo que estes possivelmente tenham relação com os despejos domésticos. 
Revista Ambientale

Revista da Universidade Estadual de Alagoas/UNEAL e-ISSN 2318-454X, Ano 13, Vol. 13 (4), 2021 https://doi.org/10.48180/ambientale.v13i4.319

Figura 4. Dendograma obtido por meio de análise de agrupamento das varáveis de qualidade de água no rio Comandaí durante o monitoramento no ponto 3.

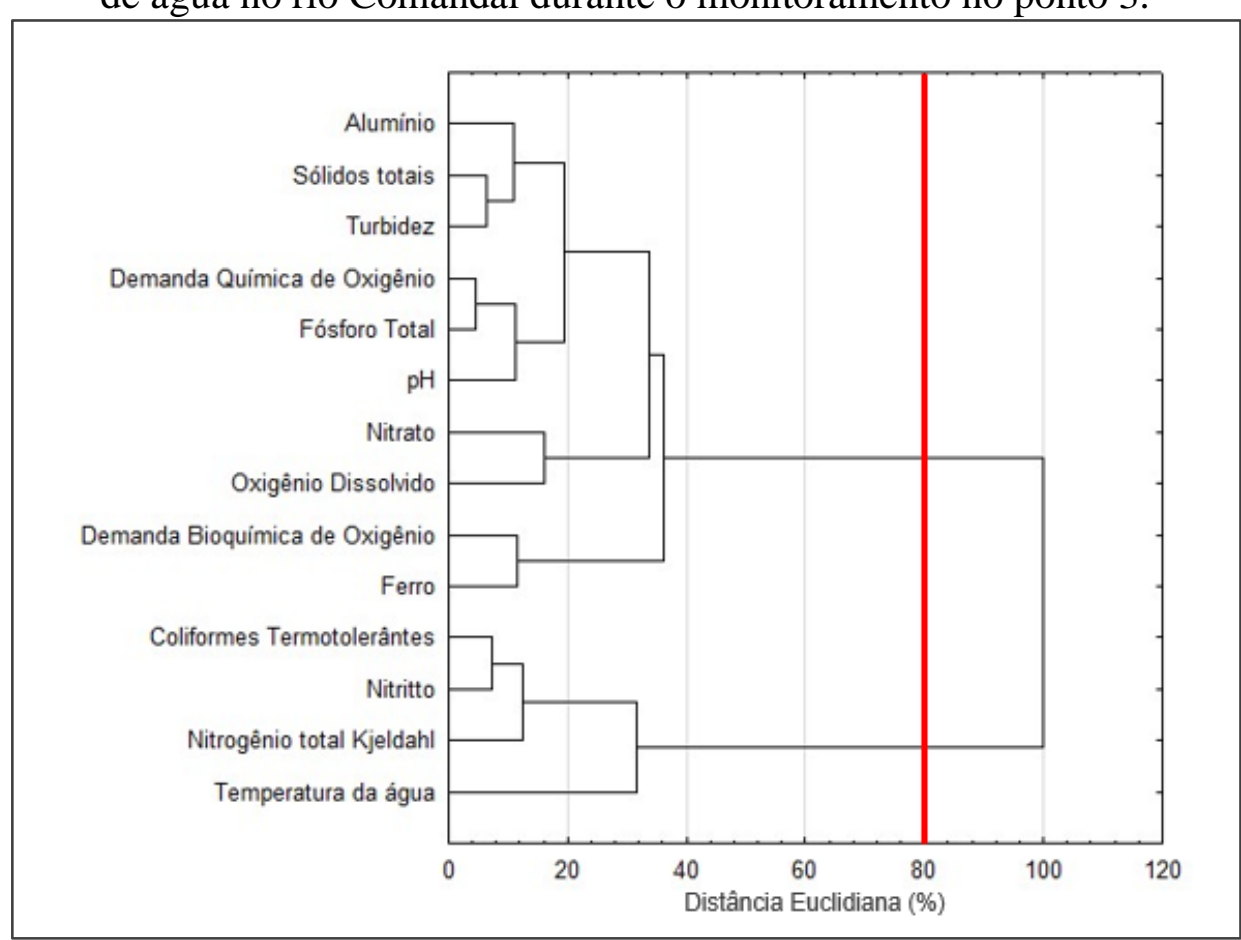

Fonte: Elaborado pelos autores.

O ponto 4, apresentou a formação de dois grupos conforme pode ser observado na Figura 5. 
Revista Ambientale

Revista da Universidade Estadual de Alagoas/UNEAL e-ISSN 2318-454X, Ano 13, Vol. 13 (4), 2021 https://doi.org/10.48180/ambientale.v13i4.319

Figura 5. Dendograma obtido por meio de análise de agrupamento das varáveis de qualidade de água no rio Comandaí durante o monitoramento no ponto 4.

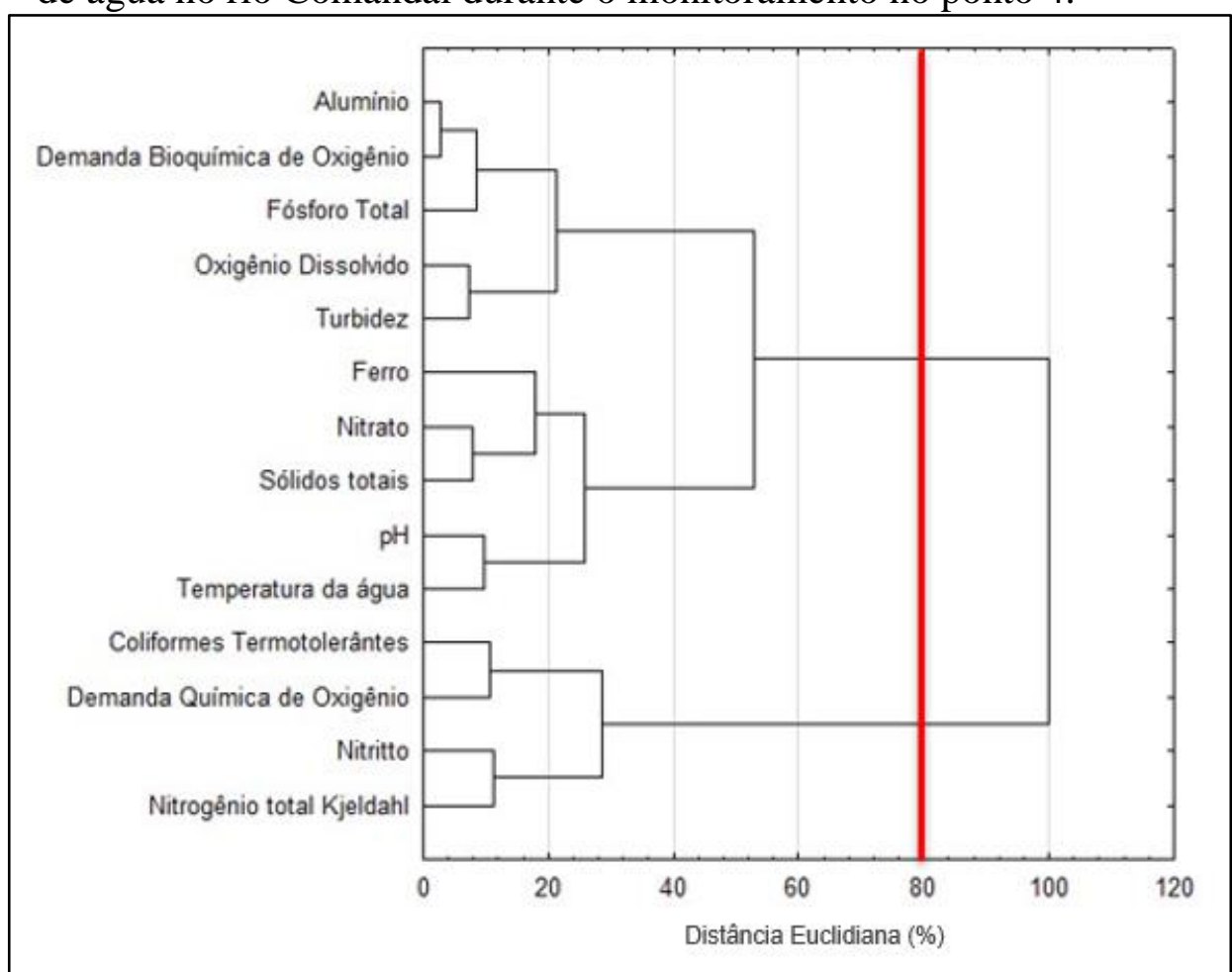

Fonte: Elaborado pelos autores.

Com os grupos formado, pode-se inferir que as possíveis fontes de poluição atreladas ao ponto 4, podem estar associadas a dissolução de rochas ou atividades agrícolas (grupo 1) e o grupo 2 aos despejos domésticos,

Por fim, com relação ao dendrograma obtido para o ponto 5 (Figura 6), observa-se que este apresentou o inverso dos demais pontos. No primeiro grupo formado pode-se inferir que ele tenha relação com despejos domésticos e ao segundo grupo, com atividades agrícolas. 
Figura 6. Dendograma obtido por meio de análise de agrupamento das varáveis de qualidade de água no rio Comandaí durante o monitoramento no ponto 5.

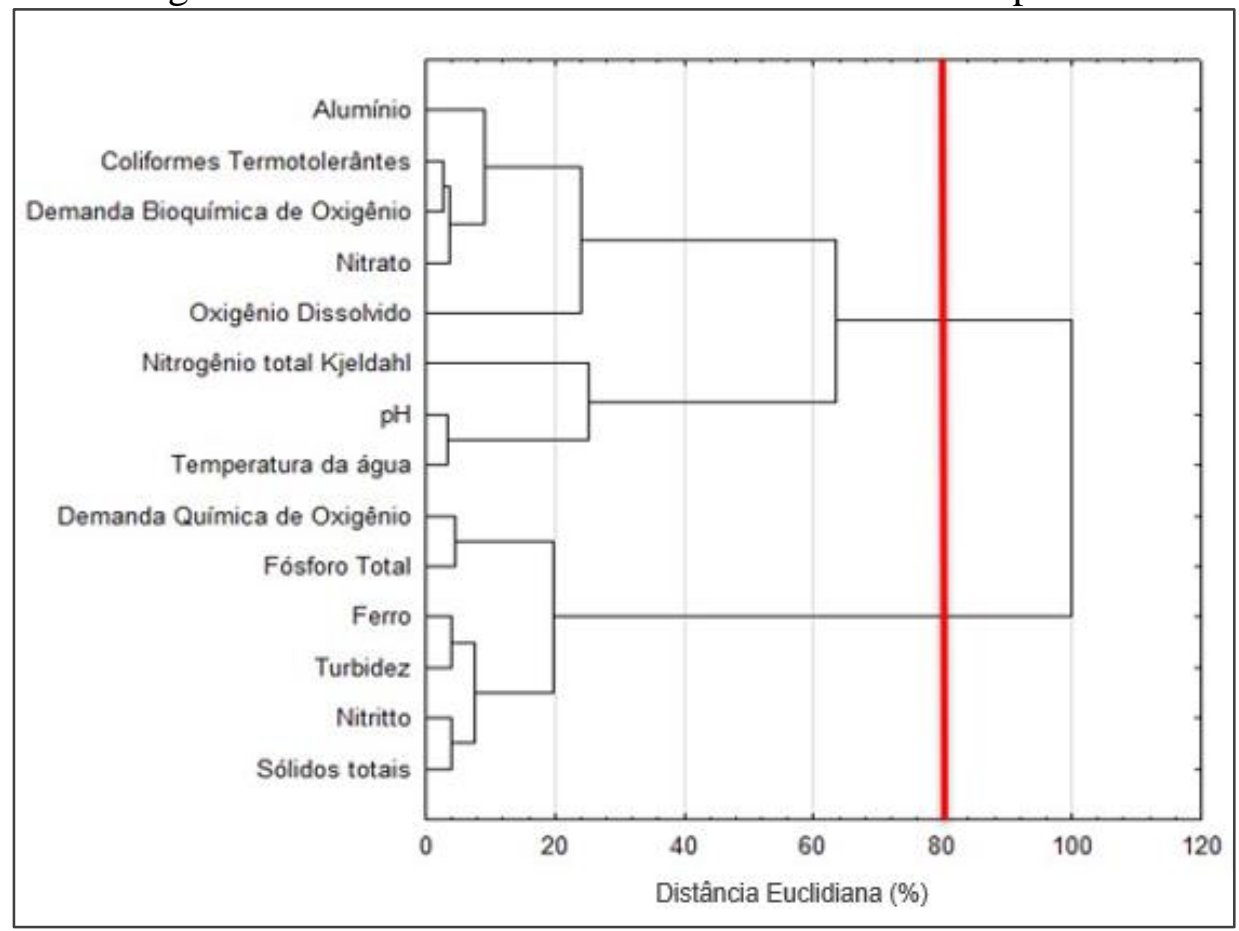

Fonte: Elaborado pelos autores.

Dos cinco pontos analisados, observa-se que quatro deles seguem um padrão semelhante, entretanto a possível fonte de poluição muda ao longo do curso de água. $\mathrm{O}$ que possivelmente torna pertinente inferir que o grupo formado com menor distância Euclidiana é a a fonte de maior impacto no ponto observado. Contudo, a distinção entre os grupos formados em cada dendrograma para cada ponto, demonstra que existe fontes de poluição difusa (SPERLING, 2014; RIBEIRO et al., 2017). Sabe-se que este manancial é fortemente influenciado por atividades agrícolas bem como pela falta de saneamento o que fica evidente nos dendrogramas formados em cada um dos pontos (FEPAM, 2019).

\section{Conclusão}

Com base neste estudo foi possível observar que os cinco pontos encontraram resultados distintos para as possíveis fontes de poluição, mesmo sendo cortados no mesmo percentual de similaridade (80\%). Isso demonstra que ao longo do rio Comandaí, há uma alteração no uso e coberturas da terra, o que modifica as fontes de poluição. Sendo assim, foi possível inferir sobre as possíveis fontes de poluição ao analisar suas similaridades por meio da análise estatística multivariada. Sugere-se para estudos futuros que sejam analisadas as mudanças no uso e cobertura do solo para o período analisado. 
Revista Ambientale

Revista da Universidade Estadual de Alagoas/UNEAL

e-ISSN 2318-454X, Ano 13, Vol. 13 (4), 2021

https://doi.org/10.48180/ambientale.v13i4.319

\section{Conflitos de interesse}

Os autores desse manuscrito não declararam conflito de interesses

\section{Referências}

AGÊNCIA NACIONAL DE ÁGUA (ANA). Panorama do enquadramento dos corpos d'água do Brasil e Panorama da qualidade das águas subterrâneas no Brasil. Brasília: ANA, 2007. Disponível em: http://portalpnqa.ana.gov.br/publicacoes.aspx. Acesso em 2019.

\section{AMERICAN PUBLIC HEALTH ASSOCIATION - APHA. Standard methods for the examination of water and wastewater. $21^{\mathrm{a}}$ ed. Washington: American Public Health Association. APHA/AWWA/WEF, 2005.}

CENTENO, Luana Nunes et al. Análise multivariada aplicada aos parâmetros de qualidade da água do rio Piratini/RS.Revista Brasileira de Engenharia e Sustentabilidade, Pelotas, v. 2, n. 2, p.45-51, dez. 2016.

\section{BRASIL. CONSELHO NACIONAL DO MEIO AMBIENTE - CONAMA. Resolução no} 357, de 17 de março de 2005. Dispõe sobre a classificação dos corpos de água e diretrizes ambientais para o seu enquadramento, bem como estabelece as condições e padrões de lançamento de efluentes e dá outras providências. Brasília-DF: MMA. 2005.

COSTA, K. A. Avaliação da qualidade da água do rio Verde, Ponta Grossa, PR, através de análise multivariada e aplicação de índices de qualidade. 2018, 87f. Dissertação (Mestrado em Química Aplicada), Universidade Estadual de Ponta Grossa, Ponta Grossa, 2018.

CRUZ, C.D. GENES - a software package for analysis in experimental statistics and quantitative genetics. Acta Scientiarum. v.35, n.3, p.271-276, 2013.

DAS, Shreya; NAG, S. K.. Application of multivariate statistical analysis concepts for assessment of hydrogeochemistry of groundwater-a study in Suri I and II blocks of Birbhum District, West Bengal, India. Applied Water Science, [s.1.], v. 7, n. 2, p.873-888, 20 jun. 2015. Springer Nature.

ESMAEILI, Somayeh et al. Multivariate statistics and hydrogeochemical modeling for source identification of major elements and heavy metals in the groundwater of Qareh-Ziaeddin plain, NW Iran. Arabian Journal Of Geosciences, [s.1.], v. 11, n. 1, p.1-14, jan. 2018. Springer Nature. 
Revista Ambientale

Revista da Universidade Estadual de Alagoas/UNEAL

e-ISSN 2318-454X, Ano 13, Vol. 13 (4), 2021

https://doi.org/10.48180/ambientale.v13i4.319

FÁVERO, L. P.; BELFIORE, P. Análise de dados: Técnicas multivariadas exploratórias com SPSS ${ }^{\circledR}$ e Stata ${ }^{\circledR}$. Rio de Janeiro: Campus Elsevier. 2015. 344 p.

FERCHICHI, Hajer et al. Understanding groundwater chemistry in Mediterranean semi-arid system using multivariate statistics techniques and GIS methods: case of Manouba aquifer (Northeastern Tunisia). Arabian Journal Of Geosciences, [s.1.], v. 10, n. 23, p.1-15, dez. 2017.

Fundação Estadual de Proteção Ambiental Henrique Roessler - FEPAM. Monitoramento da qualidade da água da região hidrográfica do Uruguai. Site oficial da FEPAM. 2019.

GLORIA, Lucivania Pereira; HORN, Bruna Carolina; HILGEMANN, Maurício. Avaliação da qualidade da água de bacias hidrográficas através da ferramenta do índice de qualidade da água - IQA. Caderno Pedagógico, [s. L.], v. 14, n. 1, p.103-119, jan. 2017.

GOMES, Maria da Conceição Rabelo et al. Similaridade de atributos físico-químicos com uso de análise multivariada aplicada à gestão qualitativa de águas subterrâneas. Geociências: UNESP, São Paulo, v. 2, n. 36, p.325-337, fev. 2017.

HAIR, F. J.; BLACK, W. C.; BABIN, B. J.; ANDERSON, R. E.; TATHAM, R. L. Análise multivariada de dados. $6^{\text {a }}$ ed. Porto Alegre: Bookman, 2009.

ISLAM, M. Atikul et al. A study of groundwater irrigation water quality in south-central Bangladesh: a geo-statistical model approach using GIS and multivariate statistics. Acta Geochimica, [s.1.], v. 37, n. 2, p.193-214, 6 jul. 2017. Springer Nature.

MAGESH, N.s.; CHANDRASEKAR, N.; ELANGO, L.. Trace element concentrations in the groundwater of the Tamiraparani river basin, South India: Insights from human health risk and multivariate statistical techniques. Chemosphere, [s.1.], v. 185, p.468-479, out. 2017. Elsevier BV.

MURTAGH, Fionn; LEGENDRE, Pierre. Ward's Hierarchical Agglomerative Clustering Method: Which Algorithms Implement Ward's Criterion?.Journal Of Classification, [s.l.], v. 31, n. 3, p.274-295, out. 2014. Springer Nature.

RIBEIRO, T. G.; et al. Estudo Da Qualidade Das Águas Por Meio Da Correlação De Parâmetros Físico-Químicos, Bacia Hidrográfica Do Ribeirão Anicuns. Geochimica Brasiliensis, v. 30, n. 1, p. 84-94, 2017.

RIO GRANDE DO SUL. Decreto n53.885, de 16 de Janeiro de 2018. Institui subdivisão das Regiões Hidrográficas do Estado do Rio Grande do Sul em Bacias Hidrográficas. Porto Alegre, RS.

SANTOS, Rosa Cecília Lima et al. Aplicação de índices para avaliação da qualidade da água da Bacia Costeira do Sapucaia em Sergipe. Engenharia Sanitária e Ambiental, [s.1.], v. 23, n. 1, p.33-46, 3 ago. 2017. FapUNIFESP. 
Revista Ambientale

Revista da Universidade Estadual de Alagoas/UNEAL e-ISSN 2318-454X, Ano 13, Vol. 13 (4), 2021

https://doi.org/10.48180/ambientale.v13i4.319

SEMA. Secretaria do Ambiente e Desenvolvimento Sustentável do Rio Grande do Sul SEMA. Bacias hidrográficas do Uruguaí. Site oficial da SEMA. 2019.

SPERLING, Marcos. Introdução à qualidade das águas e ao tratamento de esgotos. v.1, 4. ed. Belo Horizonte: Universidade Federal de Minas Gerais, 2014. 452 p.

TRINDADE, Ana Laura Cerqueira et al. Tendências temporais e espaciais da qualidade das águas superficiais da sub-bacia do Rio das Velhas, estado de Minas Gerais. Engenharia Sanitaria e Ambiental, [s.1.], v. 22, n. 1, p.13-24, 13 out. 2016. FapUNIFESP.

UNCUMUSAOğLU, Arzu Aydin; AKKAN, Tamer. Assessment of Water Quality of YağlidereStream(Turkey) Using Multivariate Statistical Techniquess. Polish Journal Of Environmental Studies, [s.1.], v. 26, n. 4, p.1715-1723, 25 jul. 2017. HARD PublishingCompany.

URBAN, Rodrigo Custodio; MANFRÉ, Luiz Augusto; SILVA, Alexandre Marco da. Multivariate statistical- and gis-based approach developed for integrated environmental analysis in urban watershed. Journal Of Urban And Environmental Engineering, [s. L.], v. 12, n. 1, p.1-5, jan. 2018.

VICINI, L; SOUZA, A. M. Análise multivariada da teoria à prática. Santa Maria: Biblioteca Central da UFSM, 2005. 215 p.

ZAMBERLAN, João Fernando et al. Índices sazonais de qualidade da água de irrigação via análise multivariada na região central do rio grande do sul. Irriga, [s.1.], v. 18, n. 3, p.376386, 8 out. 2013. Brazilian Journal of Irrigation and Drainage - IRRIGA.

ZHANG, Yunhui et al. Hydrochemical Characteristics and Multivariate Statistical Analysis of Natural Water System: A Case Study in Kangding County, Southwestern China. Water, [s.1.], v. 10, n. 1, p.01-17, 19 jan. 2018. MDPI AG. 